\title{
XVIII. On the introduction of Doppler's factor into the solution of the equations of the electron theory
}

\section{L. de la Rive}

To cite this article: L. de la Rive (1907) XVIII. On the introduction of Doppler's factor into the solution of the equations of the electron theory , Philosophical Magazine Series 6, 13:74, 279-285, DOI: $10.1080 / 14786440709463601$

To link to this article: http://dx.doi.org/10.1080/14786440709463601

巴nublished online: 16 Apr 2009.

Submit your article to this journal $\sqsubset$

Џll Article views: 4

Q View related articles ¿ 
Mr. Earhart tested four liquids-olive oil, paraffin, transformer oil, and kerosene. For the first three of these direct comparison can be made with my results.

For paraffin and transformer oil I found a breaking-down of insulation (i.e. the equivalent of a "lnee") at 175 volts and 200 volts respectively, whereas for these as for the other liquids Earhart obtained the change at 330 volts. These considerable differences may be explained by the very different conditions of the two methods.

XVIII. On the Introduction of Doppler's Factor into the Solution of the Equations of the Electron 17eory. By L. DE LA Rive*.

THE transformation of Maxwell's equations takes place as 1 follows. The equations are $\dagger:-$

$$
\begin{aligned}
& \operatorname{curl} \mathbf{D}=-\frac{1}{ \pm \pi \iota^{2}} \cdot \frac{d \mathbf{H}}{d t}, \quad \ldots . \quad . \quad . \quad . \\
& \operatorname{curl} \mathbf{H}= \pm \pi \frac{d \mathbf{D}}{d t}+4 \pi \rho \iota . . \quad . \quad . \quad .
\end{aligned}
$$

For regions of space not containing any electric charges,

$$
\operatorname{div} \mathbf{D}=0 \text {. }
$$

For a volume-distribution of electricity of density $\rho$ measured in electromagnetic units,

$$
\operatorname{div} \mathbf{D}=\rho .
$$

The distribution of $\mathbf{H}$ being solenoidal, we put

$$
\begin{aligned}
& \text { whence } \\
& \mathbf{H}=\operatorname{curl} \mathbf{A} \text {; } \\
& \operatorname{curl} \mathbf{H}=\nabla \operatorname{div} \mathbf{A}-\nabla^{2} \mathbf{A} \\
& \operatorname{curl} \frac{d \mathbf{A}}{d t}=-4 \pi r^{2} \operatorname{curl} \mathbf{D} ; \quad \text {. . . . }
\end{aligned}
$$

and, on integrating,

$$
4 \pi v^{2} \mathbf{D}=-\frac{d \mathbf{A}}{d t}-\nabla \phi, \quad . \quad . \quad . \quad .
$$

$\nabla \phi$ being a constant of integration whose curl is zero. Let the auxiliary vector $\mathbf{A}$ be arbitrarily connected with the function $\phi$ by the relation

$$
v^{2} \nabla \mathbf{A}+\frac{d \phi}{d t}=0 . \quad . \quad . \quad . \quad . \quad .
$$

* Communicated by Prof. J. H. Poynting, F.R.S.

$\uparrow$ Mathematische Einführung in die Elektronentheorie, von A. H. Bucherer, p. 76. 
Differentiating (6) with respect to $t$, we obtain

$$
4 \pi \frac{d \mathbf{D}}{d t}=-\frac{1}{v^{2}} d t^{2} \mathbf{A}-\frac{1}{v^{2}} \nabla \frac{d \phi}{d t} ; \quad . \quad . \quad .
$$

hence, using (2) and ( 8 ),

$$
\operatorname{curl} \mathrm{H}=-\frac{1}{v^{2}} \cdot \frac{d^{2} \mathbf{A}}{d t^{2}}-\frac{1}{v^{2}} \frac{\nabla d \phi}{d t}+4 \pi \rho u, \quad .
$$

and by (7)

$$
\frac{1}{v^{2}} \frac{d^{2} \mathbf{A}}{d t^{2}}-\nabla^{2} \mathbf{A}=4 \pi \rho u
$$

A similar equation may be obtained for $\phi$; and so we arrive at the system of equations:-

$$
\begin{aligned}
& \frac{1}{v^{2}} d d^{2} \mathbf{A}-\nabla^{2} \mathbf{A}=4 \pi \rho u, \quad . \quad . \quad . \quad . \quad . \\
& \frac{1}{v^{2}} \frac{d^{2} \phi}{d t^{2}}-\nabla^{2} \phi=4 \pi \rho r^{2} \\
& \mathrm{H}=\operatorname{curl} \mathbf{A} \text {, . } \\
& \mathbf{D}=\frac{1}{4 \pi v^{2}} d \mathbf{A}-\frac{1}{4 \pi v^{2}} \nabla \phi .
\end{aligned}
$$

Consider the point $\mathrm{P}_{0}$ of the field at the instant $t_{0}$, and suppose that the values of $\phi$ and $\mathbf{A}$ have been propagated to it from the electron with the velocity of light $v$. They have therefore been emitted by the electron at an instant $t$ such that the radius vector at that instant has the value

$$
r^{2}=r\left(t_{0}-t\right) . \quad \cdot \quad \cdot \cdot \cdot
$$

Let now $t_{0}$ be regarded as constant, and let $\phi$ and $\mathbf{A}$ be expressed as functions of $r$ by means of equation (6). Then

$$
\frac{d^{2} \dot{\phi}}{d t^{2}}=r^{2} d^{2} \phi, \quad \frac{d^{2} \mathbf{A}}{d t^{2}}=v^{2} \frac{d^{2} \mathbf{A}}{d r^{2}} ;
$$

and equations (I.) and (II.) become

$$
\begin{aligned}
& \frac{d^{2} \mathbf{A}}{d r^{2}}-\nabla^{2} \mathbf{A}=4 \pi \rho u, \ldots . \\
& \frac{d^{2} \phi}{d r^{2}}-\nabla^{2} \phi=4 \pi \rho v^{2} .
\end{aligned}
$$

A theorem of Beltrami's gives for a function $\phi$ of $r$ at a point $\mathrm{P}_{0}$,

$$
4 \pi \phi_{0}=\frac{1}{r}\left(\begin{array}{l}
d^{2} \phi \\
d r^{2}-\nabla^{2} \phi
\end{array}\right) d \tau
$$


the volume integral being extended throughout infinite space. Hence, by (I. $a$ ) and (II. $a$ ),

$$
\phi_{0}=\int\left(\frac{\rho v^{2}}{r}\right) d \tau,
$$

and a similar expression may be obtained for $\mathbf{A}$. In accordance with (6), each volume element of the integral should be considered at the instant $t$ defined by the relation

$$
t=t_{0}-\frac{r}{v} \text {. }
$$

Let us consider the nature of the variable $t$ defined by (6), $t_{0}$ being regarded as constant. Let the whole of space be divided up by spherical surfaces of radius $r, r$ varying from zero to infinity, corresponding to values of $t$ varying from $t_{0}$ to minus infinity. I call $t$ the time of emission; and it is evident that to a positive increment $d r$ there corresponds a negative increment $v d t$, since all the emissions are considered with respect to the same instant $t_{0}$ at $\mathrm{P}_{0}$.

On the other hand, Maxwell's equations and their transformations are directly applicable to points such as $\mathrm{P}_{0}$ of the field, which may be termed the transmission field, where the function $\phi$ and its derivatives, whether with respect to time or with respect to the space variables, are the result of the propagation of these quantities from the emitting source. Since our equations apply to the point $\mathrm{P}_{0}$, it is necessary to consider an elementary variation of $t_{0}$ and of the position of $\mathrm{P}_{0}$, the differentials concerned being denoted by $d_{0}$, $d e_{0}, d y_{0}, d z_{0}$.

Regarding both $t$ and $t$ as variable in (6), we have

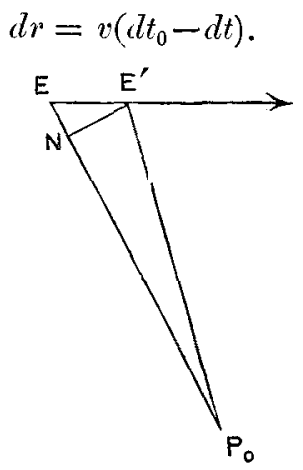

Let $\mathrm{E}$ and $\mathrm{E}^{\prime}$ be the two positions of the electron at the instants $t$ and $t+d t$ of emission. The path $\mathrm{EE}^{\prime}$ described by 
the electron is $u d t$; and on projecting it on the radius vector $\mathrm{EP}_{0}$, we have

$$
\mathrm{EN}=-d r=u d t \cos (u r),
$$

reckoning $r$ in a direction from $\mathrm{E}$ towards $\mathrm{P}_{0}$. On inserting for $d r$ its value given by (7), we find

$$
d t_{0}=d t\left(1-\frac{u t}{v} \cos (n v)\right) . \quad . \quad . \quad .
$$

I call $t_{0}$ the time of transmission; and the relation (8) shows that for a point $P_{0}$ the ratio of the elementary variations of $t$ and $t_{0}$ is constant. The ratio $\frac{d t_{0}}{d t}$, which may be termed Doppler's Factor, is the ratio of the duration of a phenomenon of emission to the duration of the same phenomenon when transmitted, such duration being supposed sufficiently short in comparison with the time required by light to travel from $\mathrm{I}$ to $\mathrm{P}_{0}$ : Its value becomes unity when the velocity of propagation is at right angles to the radius vector, since in that case the source of emission neither recedes nor approaches; and it would become zero if $u$ were equal to $v$ and the angle between the two directions were to vanish, since the source itself would then, with all its consecutive emissions, arrive at the same instant at $\mathrm{P}_{0}$.

Now that the ratio $\frac{d t_{0}}{d t}$, which we shall denote by $K$, is known and has been shown to be constant whatever the value of $r$, and that equation (II.) as well as the other equations of the system relate to the transmission space, we see that, replacing, for the sake of greater clearness, the vectorial by the cartesian expressions,

$$
\frac{1}{t^{2}} \frac{d^{2} \phi}{d t t_{0}^{2}}-\left[\frac{d^{2} \phi}{d t^{2}{ }_{0}^{2}}+\frac{d^{2} \phi}{d y_{0}{ }^{2}}+\frac{d^{2} \phi}{d z_{0}{ }^{2}}\right]=4 \pi \rho v^{2}
$$

or, putting

$$
\begin{aligned}
d t_{0}= & \mathrm{K} d t, \quad d x_{0}=\mathrm{K} d x, \quad d y_{0}=\mathrm{K} d y, \quad d z_{0}=\mathrm{K} d z, \\
& \text { 1. } d^{2} \phi \\
& v^{2} d t^{2}-\left[\frac{d^{2} \phi}{d x^{2}}+\frac{d^{2} \phi}{d y^{2}}+\frac{d^{2} \phi}{d z^{2}}\right]=\mathrm{K}^{2} 4 \pi \rho v^{2} .
\end{aligned}
$$

The first member of the equation again assumes the form which lends itself to the change of variable and the application of Beltrami's theorem, but it becomes necessary to examine whether this theorem is not modified by the change of variables. 
The volume-integral

$$
\int \frac{d^{2} \phi}{d c^{2}} d \tau
$$

in which $d x, d y, d z$ are respectively equal to $d x_{0}, d y_{0}, d \tau_{0}$ divided by $\mathrm{K}$, is identically equal to

$$
\frac{1}{\mathrm{~K}} \int \frac{d^{2} \phi}{d u_{0}^{2}} d \tau_{0}
$$

and this holds good even on replacing $\frac{d^{2} \dot{\phi}}{d} x^{2^{-}}$by $\frac{d^{2} \phi}{d \gamma^{2}}$, since $d r_{0}=\mathrm{K} d r$.

On the other hand, in the demonstration of Beltrami's theorem, as in that of an analogous theorem of Green's, the point $P_{0}$ is surrounded by a sphere whose radius tends towards zero, and the surface-integral

$$
\int \phi \frac{d\left(\begin{array}{l}
1 \\
r
\end{array}\right)}{d r} d \sigma,
$$

extended over the surface of the sphere, is considered. On substituting, as above, $d x_{0}, d y_{0}, d z_{0}$ for $d x, d y, d z$, the integral becomes

whence

$$
\frac{1}{\mathrm{~K}} \int \phi \frac{d\left(\frac{1}{r^{2}}\right)}{d r_{0}} d \sigma_{0}=\frac{1}{\mathrm{~K}} 4 \pi \phi_{0},
$$

and therefore

$$
4 \pi \phi_{0}=\mathrm{K} \int\left[\frac{d^{2} \phi}{d r^{2}}-\nabla^{2} \phi\right] d \tau,
$$

$$
\phi_{0}=\mathrm{K}^{i j} \int\left(\frac{\rho v^{2}}{r}\right) d \tau \text {. }
$$

On now replacing $d \tau$ by $d \tau_{0} / \mathrm{K}^{3}$, the factor $\mathrm{K}$ disappears. Thus, taking into account the field of emission and that of transmission, we see that the factor in question is not introduced, although it was necessary to mention this fact.

Since, however, it is necessary to complete the expression for $\phi_{0}$ by the introduction of $K$ into the denominator in order that the analytical results may accord with those obtained directly by Lorentz without the use of the notion of propagation, and with the experimental facts connected with oscillatory and circular motions of the electron, it becomes necessary to have recourse to a mechanical hypothesis involving this modification.

I believe that the hypothesis according to which the 
dimension of the electron parallel to the radius vector is modified in the desired ratio is a matter of controversy. In effect, this change of dimension is by no means justified by the subdivision of the electron into its elements of volume, each of which must correspond to an instant of emission, giving a slightly different value to the radius vector. This integration throughout the volume of the electron occurs in determining the mean radius vector, which only differs from the radius vector of the centre by a quantity of the second order.

This may be shown as follows in the case of rectilinear motion. On taking $P_{0}$ as origin and the velocity $u$ as parallel to the $x$-axis, we find, by eliminating $t_{0}-t$, the radius vector at the instant of emission expressed as a function of the coordinates of the electron at the instant $t_{0}$. This expression, in which $s$ is equal to $\left(1-u^{2} / v^{2}\right)$, is as follows *:-

$$
r=\frac{1}{s}\left[\frac{u}{v} x+\sqrt{\left(y^{2}+z^{2}\right) s+v^{2}}\right] \text {. }
$$

This corresponds to the centre of the electron; it is required to find the variation of $r$ due to the variation of the co-ordinates, and for this purpose the above expression is differentiated with respect to $x, y, z$, and then $d x, d y, d z$ are replaced by $x^{\prime}, y^{\prime}, z^{\prime}$, which represent the co-ordinates of a point in the interior of the electron relatively to its centre. This gives

$$
d r=\frac{1}{s}\left[\left[\frac{u}{v}+\frac{x}{\mathrm{D}}\right] x^{\prime}+\frac{s y}{\mathrm{D}} y^{\prime}+\frac{x z}{\mathrm{D}} z^{\prime}\right] .
$$

$x / \mathrm{D}, y / \mathrm{D}, \approx / \mathrm{D}$ are very nearly equal to the cosines of the angles made by the radius vector with the axes, since $D$ is equal to $\sqrt{\left(y^{2}+z^{2}\right) s+x^{2}}$, which is equal to the radius vector at $t_{0}$ if we suppose $s$ equal to 1 . It is required to find the mean variation of $d r$ for all points of the volume of the electron; and this is done by integrating the above expression over the entire volume and dividing by the volume. Now each term when integrated with respect to the variable which it contains gives a result which is a square and has the same value at the two limits $+l$ and $-l$ which represent the dimensions of the volume; the integral therefore vanishes, and the mean radius differs from the central one only by an infinitesimal of the second order. This is not so if $r$ is multiplied by $K$, since $u / v$ itself is not even necessarily a very small quantity of the first order.

The hypothesis which I propose as complying with plausible conditions consists in supposing that the action of the electron in motion is due to an oscillatory state of the wher, which is

* Bucherer, Einfilhrung in die Elektronentheorie, p. 84. 
propagated with the velocity of light. The ratio $d t_{v} / d t$ is thus found to be introduced as a denominator into the velocity of the oscillating mass, since Doppler's factor modifies the duration of the oscillation propagated. Now the momentum being proportional to the velocity, it is momentum in the electromagnetic field that fulfils the requirements outlined above, and this involves the assumption that field-intensity is of the nature of momentum.

In a memoir published by Bjerknes*, on the analogy between the steady electromagnetic field and the bydrodynamic field, the author shows that field-intensity in the electromagnetic field is analogous to momentum in the hydrodynamic one. There is between his conclusions, to which I would draw attention, and the necessity of introducing Doppler's factor, a concordance which is worthy of notice.

XIX. On the Thermodynamical Theory of Radiation. To the Editors of the Philosophical Magazine.

\section{Gentlemen, -}

Mr. Tuckerman's criticisms $\dagger$ appear to be based on a misunderstanding of my position. He brings as an objection to my argument, that the temperature $\mathrm{T}$ is " a quantity which is and can be defined only in terms of the properties of matter."

My argument was directed against the thermodynamical proof of the laws of radiation. It is therefore sufficient for my reductio ad absurdum that according to the thermodynamical theory underlying this proof it is possible to attribute temperature to the æther. (In the thermodynamical proof in question we are concerned with an imaginary heatengine, using xther as working substance, and working between temperatures $\mathrm{T}_{1}$ and $\mathrm{T}_{2}$.)

Apart from this, however, I wish to take this opportunity of remarking that $i t$ is quite easy to eliminate the idea of temperature completely from my original paper $\ddagger$. Let us agree to measure the warmth of the radiator by a quantity $\mathrm{E}$, which is to be equal to the average energy of a principal degree of freedom in the radiator, and therefore in the ordinary notation, to $\frac{1}{2} \mathrm{RT}$.

The radiation-function $\phi$ must be a function of $\lambda, \mathrm{E}, \mathrm{V}, e$, $m$, and $\mathrm{K}$. There are now six independent quantities and four independent physical units, namely those of length, mass, time, and inductive capacity. As before, we can combine the six quantities in two independent ways so as to

* Archives des Sciences phys. et nat. 1905, t. xx. p. 325.

† Phil. Mag. Nov. 1906, p. 498.

$\ddagger$ Proc. Roy. Soc. A. Ixxví. p. 545.

Plit. Mag. S. 6. Vol. 13. No. 74. Feb. 1907. 